\title{
Secondary lepton distributions as a probe of the top-Higgs coupling at the LHC
}

\author{
K. Kołodziej \\ Institute of Physics, University of Silesia, \\ ul. Uniwersytecka 4, PL-40007 Katowice, Poland \\ E-mail: karol.kolodziej@us.edu.pl
}

ABSTRACT: The differential distributions in rapidity and angles of the secondary lepton in the associated production of the top quark pair and higgs boson in proton-proton collisions at the LHC are quite sensitive to the top-higgs coupling. However, the effects of anomalous couplings of the most general $t \bar{t} h$ interaction with operators of dimension-six that are clearly visible in the signal of the associated production of the top quark pair and higgs boson are to large extent obscured by the background sub-processes with the same final state. This means that analyses of such effects, in addition to higher order corrections that are usually calculated for the on-shell top quarks and higgs boson, should include their decays and possibly complete off resonance background contributions to the corresponding exclusive reactions.

Keywords: Monte Carlo Simulations, Hadronic Colliders

ARXIV EPRINT: 1303.4962 


\section{Contents}

1 Introduction 1

2 Calculation details 2

$\begin{array}{lll}3 & \text { Results } & 4\end{array}$

$\begin{array}{llr}4 & \text { Summary and conclusions } & 8\end{array}$

\section{Introduction}

Associated production of the top quark pair and higgs boson was proposed as a sensitive probe of the top-higgs Yukawa coupling $g_{t t h}$ at the $e^{+} e^{-}$linear collider (LC) [1-5] more than 20 years ago $[6,7]$. A clean experimental environment of the $\mathrm{LC}$ seems to be the best place to study the higgs boson profile, including the measurement of $g_{t t h}$, but the project of LC is still at the rather early stage of TDR. Fortunately, the top quarks are copiously produced at the LHC that, among others, allows for more and more precise determination of the top quark pair production cross section and for measurements of the cross sections of $t \bar{t}+$ jets, see [8] for a review. The measurement of production of $t \bar{t}+b \bar{b}[9]$ is particularly interesting, as it is relevant for observation of the associated production of the top quark pair and higgs boson, with the higgs decaying into $b \bar{b}$. The latter should be the dominant decay mode, if the new boson at a mass of about $125 \mathrm{GeV}$ observed at the LHC $[10,11]$ is indeed the higgs.

The associated production of the top quark pair and higgs boson in the proton-proton collisions at the LHC

$$
p p \rightarrow t \bar{t} h
$$

is dominated by the gluon-gluon fusion mechanism. Taking into account decays: $h \rightarrow b \bar{b}$, $t \rightarrow b W^{+}, \bar{t} \rightarrow \bar{b} W^{+}$and the subsequent decays of the $W$-bosons, one should consider hard scattering partonic processes as, e.g.,

$$
g g \rightarrow b u \overline{d b} \mu^{-} \bar{\nu}_{\mu} b \bar{b}
$$

corresponding to one of the $W$ 's decaying hadronically and the other leptonically. Reaction (1.2) receives contributions from 67300 Feynman diagrams in the leading order of the standard model (SM), in the unitary gauge neglecting masses smaller than $m_{b}$, of which barely 56 diagrams constitute the signal of the $t \bar{t} h$ production and subsequent decay. Some examples of the Feynman diagrams of (1.2) are shown in figure 1. The 56 signal diagrams are obtained from those depicted in figure 1(a), 1(b) and 1(c) by attaching the $h b \bar{b}$-vertex 


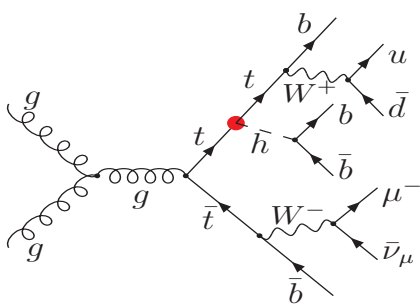

(a)

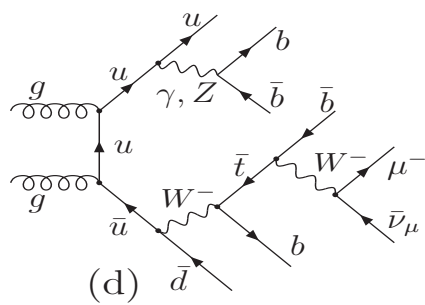

(d)

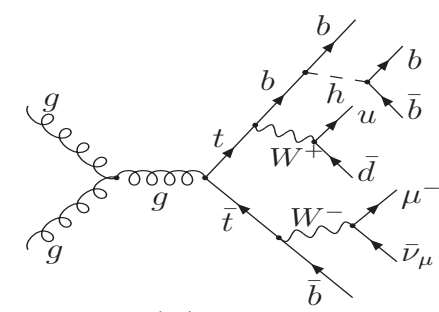

(b)

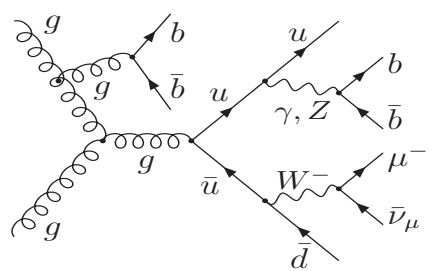

(e)

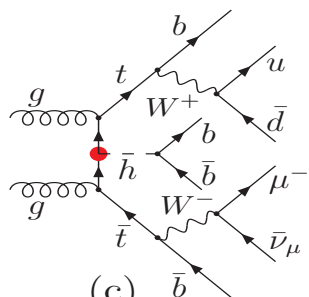

(c)

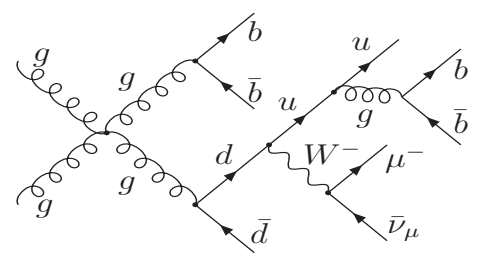

(f)

Figure 1. Examples of the lowest order Feynman diagrams of reaction (1.2): (a), (b) and (c) are the signal diagrams of $t \bar{t} h$ production, (d), (e) and (f) are the $t \bar{t} h$ background contributions. Blobs indicate the higgs-top coupling.

to other top or bottom quark lines, interchanging external $b$ and $\bar{b}$ quarks in each of the figures and interchanging the two initial state gluons of figure 1(c). The diagrams shown in figure 1(d), 1(e) and 1(f) are just a few examples of the background contributions to associated production of the higgs boson and top quark pair.

A question arises whether the associated production of the higgs boson and top quark pair can be sensitive to possible modifications of the SM top-higgs Yukawa coupling or not. The question will be addressed in this work by showing how the distributions of the secondary lepton are changed in the presence of such modifications. The distributions computed with the signal diagrams only will be compared with those computed with the full set of the leading order Feynman diagrams that will demonstrate how the background contributions obscure relatively clear effects of the anomalous $t \bar{t} h$ coupling in the signal cross section. Although the issue may seem somewhat premature from the experimental side, but in view of the excellent performance of the LHC, it may become relevant in quite a near future.

\section{Calculation details}

The calculation is performed in a fully automatic way with a new version [12] of carlomat [13, 14], a general purpose program for Monte Carlo computation of lowest order cross sections. The most general Lagrangian of $t \bar{t} h$ interaction including corrections from dimension-six operators that has been implemented in the program has the following form $[15]$

$$
\mathcal{L}_{t \bar{t} h}=-g_{t \bar{t} h} \bar{t}\left(f+i f^{\prime} \gamma_{5}\right) t h,
$$

where $g_{t \bar{t} h}=m_{t} / v$, with $v=\left(\sqrt{2} G_{F}\right)^{-1 / 2} \simeq 246 \mathrm{GeV}$, is the top-higgs Yukawa coupling. The couplings $f$ and $f^{\prime}$ are assumed to be real. They describe, respectively, scalar and 
pseudoscalar departures from the purely scalar top-higgs Yukawa coupling of SM. The latter is reproduced for $f=1$ and $f^{\prime}=0$. Other dimension-six gauge-invariant effective operators that may have affected the $t \bar{t} h$ interaction are redundant, in a sense that they can be eliminated with the use of the equations of motion, both for the on- and off-shell particles [15]. Obviously, the process of associated production of the higgs boson and top quark pair will be affected by many other possible deviations from the SM couplings. They are not considered here, as the primary goal of the present work is to illustrate just the effects of the anomalous $t \bar{t} h$ interaction on the distributions of the secondary lepton. However, some deviations, eg., the anomalous $W t b$ coupling generated by the gauge-invariant dimension-six effective operators, which is present in the $t \bar{t} h$ signal diagrams of figures 1(a)(c) and in some off resonance background diagrams such as the one depicted in figure 1(d), can be easily included, as it has been already implemented in carlomat. See [16] for the illustration of some effects on the top quark pair production at the LHC that can be caused by the $W t b$ coupling.

The couplings $f$ and $f^{\prime}$ of Lagrangian (2.1) belong to least constraint couplings of the SM. For the higgs boson with a mass of $125 \mathrm{GeV}$, practically the only model independent way to constrain them is to measure the $t \bar{t} h$ production $[17,18]$. First results of search for this process in $p p$ collisions at the LHC are reported in [19-21]. Indirect constraints of the $t \bar{t} h$ interaction vertex can be derived from measurements of the higgs boson production rate through the gluon-gluon fusion process, which is dominated by a top-quark loop, and of the higgs boson decay into 2 photons that, despite being dominated by the $W$ boson loop, also receives a significant contribution from the top-quark loop. However, extraction of the $t \bar{t} h$ coupling in this way relies on the assumption that the loops do not receive contributions from new massive fundamental particles beyond those of the SM. If two universal scale factors are assumed, one for the higgs boson Yukawa couplings to all the SM fermion species and the other for the higgs boson couplings to the EW gauge bosons, and if there is no new physical degrees of freedom, then the scalar coupling $f$ of eq. (2.1) can be constraint at $95 \%$ C.L. to be in the following regions:

$$
\begin{array}{ll}
f \in[-1.2,-0.6] \cup[0.6,1.3] & \text { ATLAS }[22] \\
f \in[0.3,1.0] & \text { CMS }[23] .
\end{array}
$$

It should be noted at this point that an opposite sign of the higgs boson coupling to fermions with respect to its coupling to the gauge bosons is required in the Lagrangian for the unitarity and renormalizability of the theory [26] and vacuum stability [27]. Therefore, the interval in the range of negative numbers in (2.2) is highly disfavoured. The relative sign of both couplings could probably be best determined in the reaction of associated production of the top quark and higgs boson in proton-proton collisions at the LHC through the underlying $t$-channel partonic process $q b \rightarrow t q^{\prime} h[24,25]$.

In carlomat, the on-shell poles in propagators of unstable particles, both the $s$ - and $t$-channel ones, are avoided by making the following substitutions:

$$
m_{b}^{2} \rightarrow M_{b}^{2}=m_{b}^{2}-i m_{b} \Gamma_{b}, \quad b=Z, W, h, \quad m_{t} \rightarrow M_{t}=\sqrt{m_{t}^{2}-i m_{t} \Gamma_{t}}
$$


where the particle widths are assumed to be constant and the square root with positive real part is chosen, see $[13,14]$ for details. In order to minimize unitarity violation effects at high energies caused by substitutions (2.4), which correspond to re-summation of one particle irreducible higher order contributions to $s$-channel propagators, the computation is performed in the complex mass scheme, where the electroweak (EW) couplings are parametrized in terms the complex EW mixing parameter $\sin ^{2} \theta_{W}=1-M_{W}^{2} / M_{Z}^{2}$ which preserves the lowest order Ward identities $[28,29]$. Note, that the electric charge $e_{W}$ can be defined as a real quantity in terms of $\alpha_{W}$

$$
e_{W}=\sqrt{4 \pi \alpha_{W}}, \quad \text { with } \quad \alpha_{W}=\frac{\sqrt{2} G_{F} m_{W}^{2}}{\pi}\left(1-\frac{m_{W}^{2}}{m_{Z}^{2}}\right),
$$

as it enters all the EW couplings multiplicatively, which is our choice in the present work. The only effect of using the complex masses of (2.4) in eq. (2.5) would be the overall change of normalization of the cross section. The top-higgs Yukawa coupling is defined in the complex mass scheme by

$$
g_{t \bar{t} h}=e_{W} \frac{M_{t}}{2 \sin \theta_{W} M_{W}},
$$

i.e., it is a complex quantity, as it is parametrized in terms of the complex masses of (2.4) and complex EW mixing parameter $\sin \theta_{W}$.

\section{Results}

In this section some results for the differential cross sections and distributions of reaction

$$
p p \rightarrow b \bar{b} b u \overline{d b} \mu^{-} \bar{\nu}_{\mu}
$$

at $\sqrt{s}=14 \mathrm{TeV}$ are presented. For the sake of simplicity and easy reproducibility of the results, only one hard scattering process (1.2) that dominates at that energy is taken into account. It is folded with CTEQ6L parton distribution functions [30] at the scale $Q=2 m_{t}+m_{h}$.

The initial physical input parameters used in the computation are the following. The strong coupling between quarks and gluons is given by $g_{s}=\sqrt{4 \pi \alpha_{s}}$, with $\alpha_{s}\left(m_{Z}\right)=0.118$. The EW couplings are parametrized in terms of the electric charge of (2.5) that is kept real and the complex EW mixing parameter $\sin \theta_{W}$, as described in section 2, with the EW gauge boson masses and widths: $m_{W}=80.419 \mathrm{GeV}, \Gamma_{W}=2.12 \mathrm{GeV}, m_{Z}=91.1882 \mathrm{GeV}$, $\Gamma_{Z}=2.4952 \mathrm{GeV}$ and the Fermi coupling $G_{F}=1.16639 \times 10^{-5} \mathrm{GeV}^{-2}$. The top quark and higgs boson masses are: $m_{t}=173 \mathrm{GeV}, m_{h}=125 \mathrm{GeV}$ and their widths that are calculated to the lowest order of SM are the following: $\Gamma_{t}=1.49165 \mathrm{GeV}, \Gamma_{h}=4.9657 \mathrm{MeV}$. The $b-$ quark and muon masses are also kept non zero, but their actual values: $m_{b}=4.5 \mathrm{GeV}$ and $m_{\mu}=105.65837 \mathrm{MeV}$, are numerically irrelevant in practise. Masses of the light quarks of (3.1) are neglected.

Jets are identified with their original partons and the following cuts on the transverse momenta $p_{T}$, pseudorapidities $\eta$, missing transverse energy $\mathbb{E}^{T}$ and separation $\Delta R_{i k}$ in the 
pseudorapidity-azimuthal angle $(\varphi)$ plane between the objects $i$ and $k$ are imposed:

$$
\begin{array}{ll}
p_{T l}>30 \mathrm{GeV}, & p_{T j}>30 \mathrm{GeV}, \quad\left|\eta_{l}\right|<2.1, \quad\left|\eta_{j}\right|<2.4, \\
E^{T}>20 \mathrm{GeV}, & \Delta R_{l j, j j}=\sqrt{\left(\eta_{i}-\eta_{k}\right)^{2}+\left(\varphi_{i}-\varphi_{k}\right)^{2}}>0.4,
\end{array}
$$

where the subscripts $l$ and $j$ stand for lepton and jet. Cuts (3.2) should allow to select events with separate jets, an isolated charged lepton and missing transverse momentum.

Moreover, $100 \%$ efficiency of $b$ tagging is assumed and events of the associated production of top quark pair and higgs boson in reaction (3.1) are selected by imposing the following invariant mass cuts: on the invariant mass of two non $b$ jets, $b_{\sim b_{1}}$ and $b_{\sim b_{2}}$,

$$
60 \mathrm{GeV}<\left[\left(p_{\sim b_{1}}+p_{\sim b_{2}}\right)^{2}\right]^{1 / 2}<90 \mathrm{GeV},
$$

on the transverse mass of the muon-neutrino system

$$
\left[m_{\mu}^{2}+2\left(m_{\mu}^{2}+\left|\vec{p}_{\mu}^{T}\right|^{2}\right)^{1 / 2}\left|\not{p}^{T}\right|-2 \vec{p}_{\mu}^{T} \cdot \not{p}^{T}\right]^{1 / 2}<90 \mathrm{GeV}
$$

on the invariant mass of a $b$ jet, $b_{1}$, and the two non $b$ jets

$$
\left|\left[\left(p_{b_{1}}+p_{\sim b_{1}}+p_{\sim b_{2}}\right)^{2}\right]^{1 / 2}-m_{t}\right|<30 \mathrm{GeV},
$$

on the transverse mass $m_{T}$ of a $b$ quark, $b_{2}$, muon and missing transverse energy

$$
m_{t}-30 \mathrm{GeV}<m_{T}<m_{t}+10 \mathrm{GeV}
$$

and the invariant mass cut on two $b$ jets, $b_{3}$ and $b_{4}$,

$$
\left|\left[\left(p_{b_{3}}+p_{b_{4}}\right)^{2}\right]^{1 / 2}-m_{h}\right|<m_{b b}^{\text {cut }}
$$

with either $m_{b b}^{\text {cut }}=20 \mathrm{GeV}$ or, more optimistically, $m_{b b}^{\text {cut }}=10 \mathrm{GeV}$. In (3.6), $m_{T}$ is the transverse mass defined by

$$
m_{T}^{2}=m^{2}+2\left(m^{2}+\left|\vec{p}_{b_{2}}^{T}+\vec{p}_{\mu}^{T}\right|^{2}\right)^{1 / 2} \mathbb{E}^{T}-2\left(\vec{p}_{b_{2}}^{T}+\vec{p}_{\mu}^{T}\right) \cdot \vec{p}^{T}
$$

with $m$ being the invariant mass of the $b-\mu$ system given by $m^{2}=\left(p_{b_{2}}+p_{\mu}\right)^{2}$. Cuts (3.3)(3.6) should allow to identify the secondary $W$ bosons, the top quarks and the higgs boson. They were used before in the context of the associated production of the top quark pair and higgs boson in $e^{+} e^{-}$collisions at the LC [31, 32].

For the sake of illustration, the $t \bar{t} h$ couplings of (2.1) are assigned the following values: $f=1,0$ and $f^{\prime}=0, \pm 1$ and the differential distributions of the final state muon, generally referred to as lepton, of reaction (3.1) are computed, first with the 56 signal Feynman diagrams of the associated production of the top quark pair and higgs boson and then with the complete set of 67300 Feynman diagrams, as discussed in section 1. The rapidity and angular differential cross sections and distributions of the lepton for which the effects 
of anomalous couplings are best visible will be shown in figures 3-6 and the distributions in the lepton transverse momentum or energy which are practically not affected by the couplings will not be presented.

The size of background contributions to the associated production of the higgs boson and top quark pair in $p p$ collisions at $\sqrt{s}=14 \mathrm{TeV}$ is illustrated in figure 2, where the differential cross sections of (3.1) are plotted as functions of the muon rapidity, $y_{l}$, cosine of the muon angle with respect to beam, $\cos \theta_{l \mathrm{~b}}$ and $\operatorname{cosine}$ of the muon angle with respect to the reconstructed higgs boson momentum, $\cos \theta_{l h}$. The cross sections plotted in figure 2 have been computed with different cuts. In the left and central panel, the boxes shaded in light grey show the cross sections computed with cuts (3.2) and the grey boxes depict the cross sections calculated with cuts (3.2) and the invariant mass cuts (3.3)-(3.6). These results are not shown in the right panel of figure 2 , as it is in principle not possible to reconstruct the higgs boson momentum without cut (3.7) on its invariant mass. The shortdashed (dotted) lines show the results for $m_{b b}^{\text {cut }}=20 \mathrm{GeV}\left(m_{b b}^{\text {cut }}=10 \mathrm{GeV}\right)$ and the solid line in each panel of Fig 2 shows the results for the signal cross section calculated with $m_{b b}^{\text {cut }}=20 \mathrm{GeV}$. It should be noted here that the signal cross section is fairly independent of $m_{b b}^{\text {cut }}$. As can be seen, the background is quite large and decreasing a value of $m_{b b}^{\text {cut }}$ seems to be a right way towards its reduction.

Distributions in 3 different kinematical variables of the final state lepton of (3.1): rapidity, $y_{l}$, cosine of the angle with respect to the beam, $\cos \theta_{l \mathrm{~b}}$ and cosine of the angle with respect to the reconstructed higgs boson momentum, $\cos \theta_{l h}$ are shown in figures 35 . In each of the figures, the left panels show the $t \bar{t} h$ production signal, computed with 56 Feynman diagrams, and the right panels show the complete leading order predictions, computed with 67300 Feynman diagrams. The grey boxes show the corresponding SM results, i.e. the results obtained with $f=1$ and $f^{\prime}=0$. Cuts (3.2)-(3.6) and (3.7) with $m_{b b}^{\text {cut }}=20 \mathrm{GeV}$ are applied to all the distributions presented. A relatively clear effect of the anomalous couplings $f$ and $f^{\prime}$ that can be seen in the $t \bar{t} h$ signal distributions on the left hand side of all figures 3-6 is to large extent obscured by the background contributions in the plots on the right hand side which show the full leading order results.

In order to illustrate what a role invariant mass cuts (3.2)-(3.7) play, the distributions of the final state lepton of (3.1) at $\sqrt{s}=14 \mathrm{TeV}$ in $y_{l}$ and $\cos \theta_{l \mathrm{~b}}$ with different cuts are compared in figure 6 . The left panels show the distributions with the cuts given by (3.2) and the right panels show the distributions with cuts (3.2)-(3.6) and (3.7) with $m_{b b}^{\text {cut }}=10 \mathrm{GeV}$ for two different combinations of the scalar and pseudoscalar $t \bar{t} h$ couplings of (2.1). Again the grey boxes show the SM results. Although the invariant mass cuts suppress the background contributions to some extent, the degree of suppression does not look very satisfactory.

The signal significance $\mu=\sigma\left(f, f^{\prime}\right) / \sigma_{\mathrm{SM}}$ and corresponding differences in expected numbers of events, $\Delta n=n\left(f, f^{\prime}\right)-n_{\mathrm{SM}}$, for different combinations of the couplings $\left(f, f^{\prime}\right)$, with the $\mu^{-}$in the forward $\left(\cos \theta_{l h}>0\right)$ or backward $\left(\cos \theta_{l h}<0\right)$ hemisphere with respect to the direction of the higgs boson, in reaction (3.1) at $\sqrt{s}=14 \mathrm{TeV}$ are shown in table 1 . The cuts are given by $(3.2)-(3.7)$ with $m_{b b}^{\text {cut }}=20 \mathrm{GeV}$ (columns $\left.2-5\right)$ and $m_{b b}^{\text {cut }}=10 \mathrm{GeV}$ (columns 6-9). The event numbers have been calculated assuming an integrated luminosity 

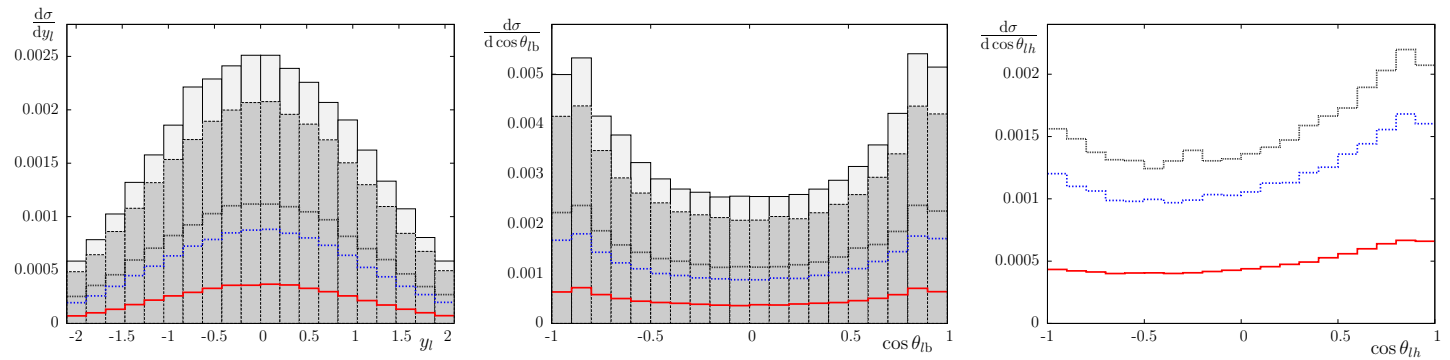

Figure 2. The differential cross sections of (3.1) at $\sqrt{s}=14 \mathrm{TeV}$ as functions of the muon rapidity $y_{l}$, cosine of the muon angle with respect to beam $\cos \theta_{l \mathrm{~b}}$ and cosine of the muon angle with respect to the higgs boson $\cos \theta_{l h}$ computed with different cuts as described in the main text.
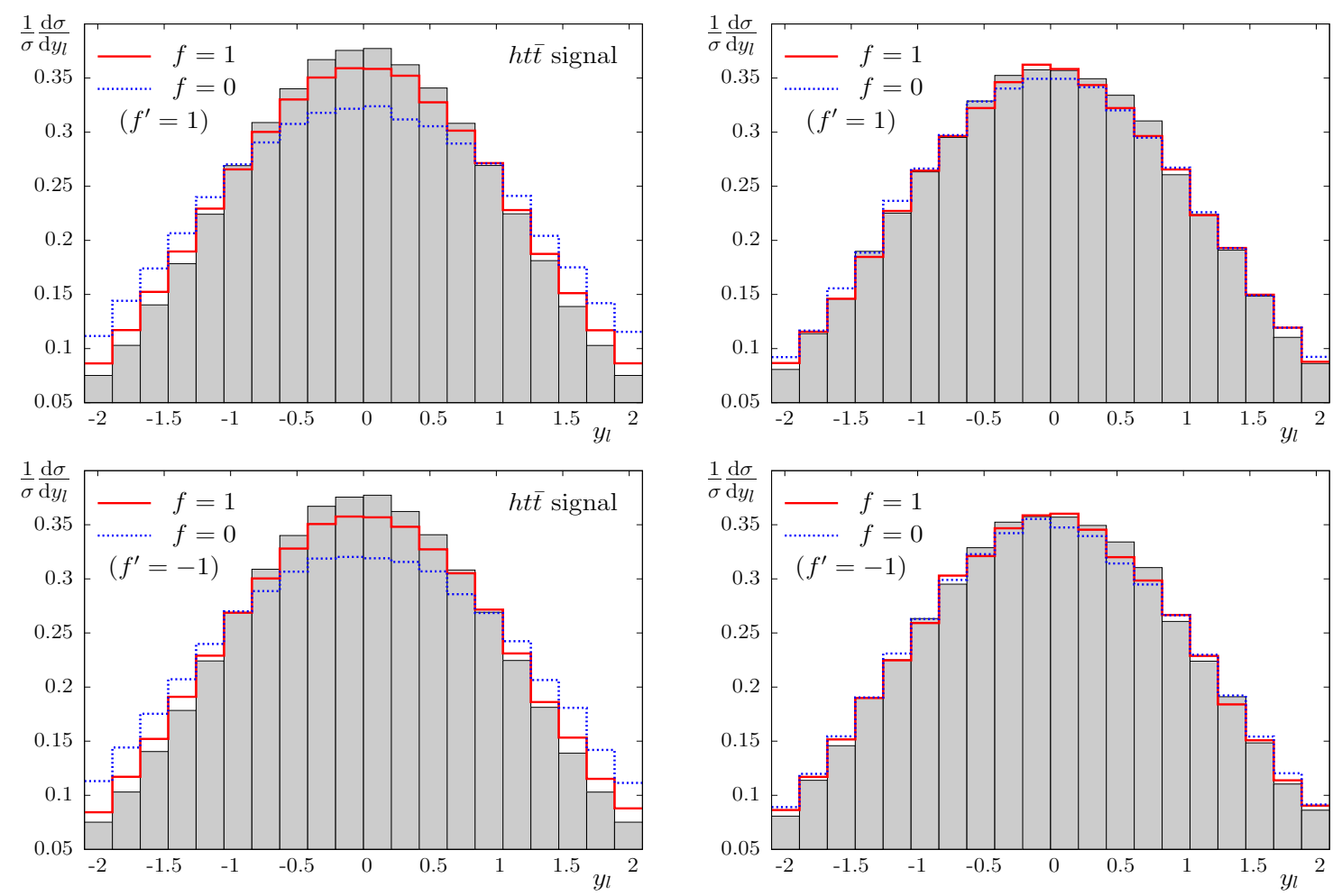

Figure 3. Distributions in rapidity of the final state lepton of (3.1) in $p p$ collisions at $\sqrt{s}=14 \mathrm{TeV}$ with different combinations of the scalar and pseudoscalar $t \bar{t} h$ couplings: $t \bar{t} h$ production signal (left panels) and complete leading order prediction (right panels).

of $100 \mathrm{fb}^{-1}$ and $100 \%$ detection efficiency. Therefore, they should be treated with care, in particular because of the fact that only the leading order contributions to reaction (3.1) are taken into account. However, the leading order predictions for signal significance $\mu$ are more reliable. In particular, $\mu \approx 1.2$ for $f=1$ and $\left|f^{\prime}\right|=1$ indicates a potential of the reaction of associated production of the higgs boson and top quark pair in obtaining direct limits on the pseudoscalar coupling $f^{\prime}$. If only the $t \bar{t} h$ signal contributions to the cross section are taken into account, then the signal significance for this particular combination of couplings becomes even bigger, amounting to $\mu=1.4$ in the forward and $\mu=1.6$ in the 

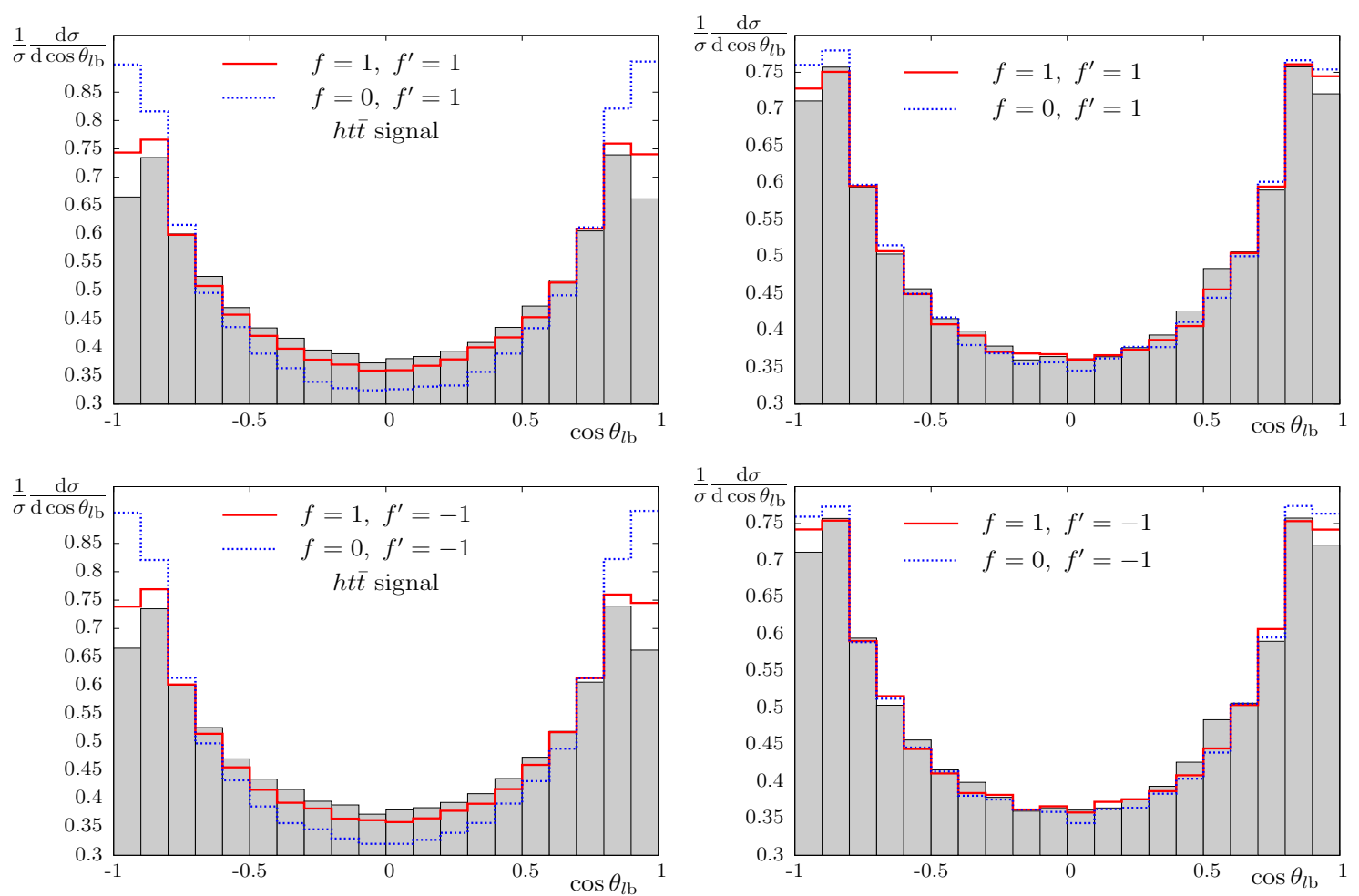

Figure 4. Distributions in cosine of the angle between the final state lepton of (3.1) with respect to the beam in $p p$ collisions at $\sqrt{s}=14 \mathrm{TeV}$ with different combinations of the scalar and pseudoscalar $t \bar{t} h$ couplings: $t \bar{t} h$ production signal (left panels) and complete leading order prediction (right panels).

\begin{tabular}{|c|rrrr|rrrr|}
\hline & \multicolumn{4}{|c|}{$m_{b b}^{\text {cut }}=20 \mathrm{GeV}$} & \multicolumn{4}{c|}{$m_{b b}^{\text {cut }}=10 \mathrm{GeV}$} \\
& $\cos \theta_{l h}<0$ & \multicolumn{1}{c|}{$\cos \theta_{l h}>0$} & \multicolumn{1}{c|}{$\cos \theta_{l h}<0$} & \multicolumn{1}{c|}{$\cos \theta_{l h}>0$} \\
\cline { 2 - 10 }$\left(f, f^{\prime}\right)$ & $\mu$ & $\Delta n$ & $\mu$ & $\Delta n$ & $\mu$ & $\Delta n$ & $\mu$ & $\Delta n$ \\
\hline$(0,1)$ & 0.90 & -148 & 0.83 & -265 & 0.85 & -174 & 0.78 & -275 \\
$(0,-1)$ & 0.90 & -151 & 0.84 & -252 & 0.84 & -188 & 0.78 & -270 \\
$(1,1)$ & 1.20 & 295 & 1.17 & 251 & 1.23 & 261 & 1.17 & 210 \\
$(1,-1)$ & 1.20 & 302 & 1.15 & 238 & 1.24 & 279 & 1.18 & 221 \\
\hline
\end{tabular}

Table 1. The signal significance $\mu$ and corresponding difference in numbers of events $\Delta n$ for different combinations of the couplings $\left(f, f^{\prime}\right)$ in reaction $(3.1)$ at $\sqrt{s}=14 \mathrm{TeV}$.

backward hemisphere with respect to the direction of the higgs boson. This again shows how the off resonance background contributions obscure the signal of $t \bar{t} h$ production.

\section{Summary and conclusions}

The differential cross sections and distributions of the final state lepton of (3.1) in rapidity, cosine of its angle with respect to the beam and cosine of its angle with respect to the reconstructed higgs boson momentum have been computed to the leading order in the 

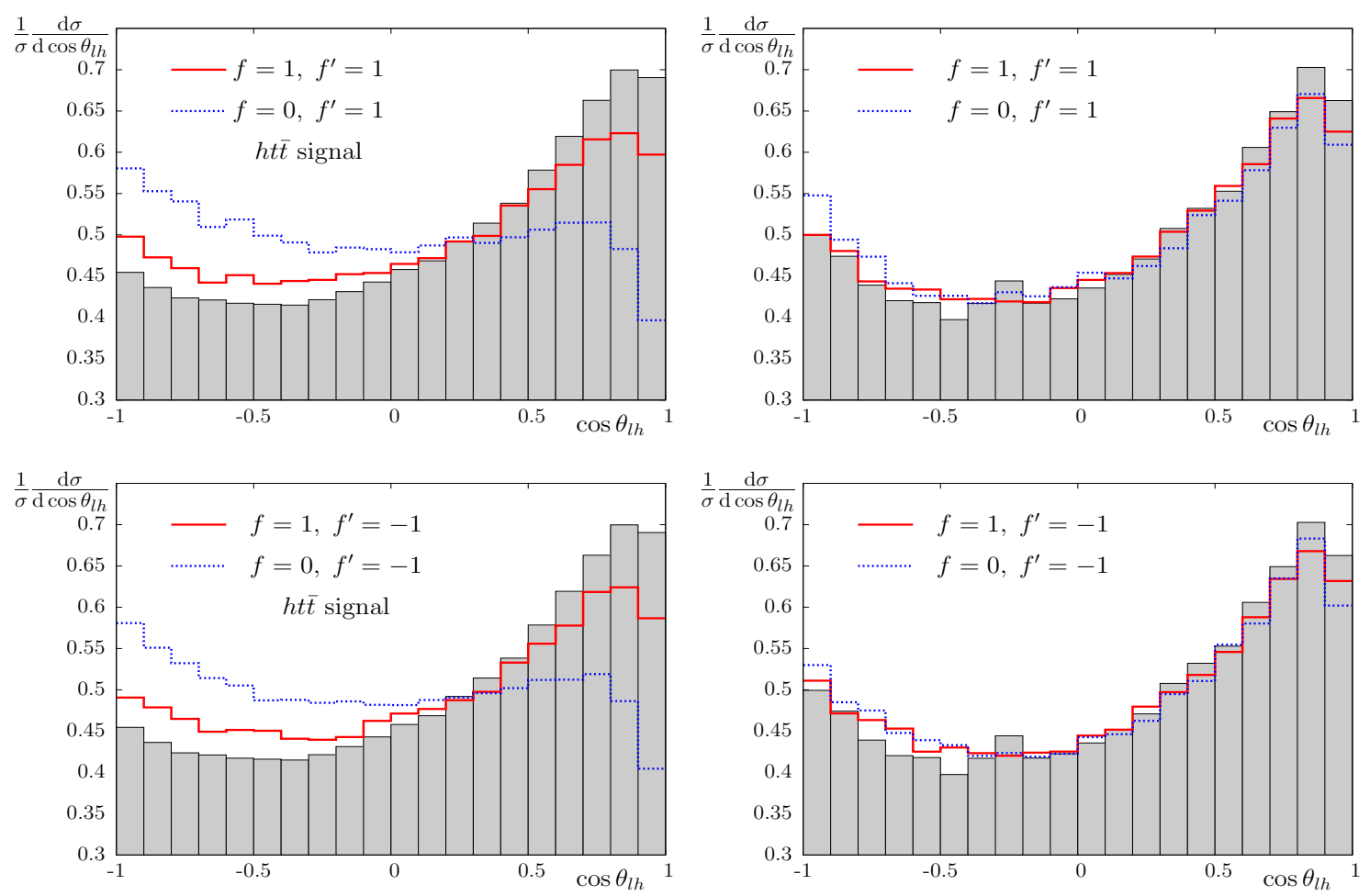

Figure 5. Distributions in cosine of the angle between the final state lepton of (3.1) and the higgs boson in $p p$ collisions at $\sqrt{s}=14 \mathrm{TeV}$ with different combinations of the scalar and pseudoscalar $t \bar{t} h$ couplings: $t \bar{t} h$ production signal (left panels) and complete leading order prediction (right panels).

presence of most general $t \bar{t} h$ interaction with operators of dimension-six. The distributions computed with the $t \bar{t} h$ signal diagrams only, which are substantially changed in the presence of anomalous $t \bar{t} h$ couplings, have been compared with those computed with the full set of the leading order Feynman diagrams. The comparison have shown that the background contributions to large extent obscure the relatively clear effects of the anomalous $t \bar{t} h$ coupling in the signal distributions. This means that analyses of such effects [33], in addition to higher order corrections [34] that are usually calculated for the on-shell top quarks and higgs boson, should include their decays and possibly complete off resonance background contributions to the corresponding exclusive reactions. The only reasonable way to make the effects of anomalous couplings better visible seems to be imposing more and more restrictive cuts.

\section{Acknowledgments}

This project was supported in part with financial resources of the Polish National Science Centre (NCN) under grant decision number DEC-2011/03/B/ST6/01615 and by the Research Executive Agency (REA) of the European Union under the Grant Agreement number PITN-GA-2010-264564 (LHCPhenoNet). 

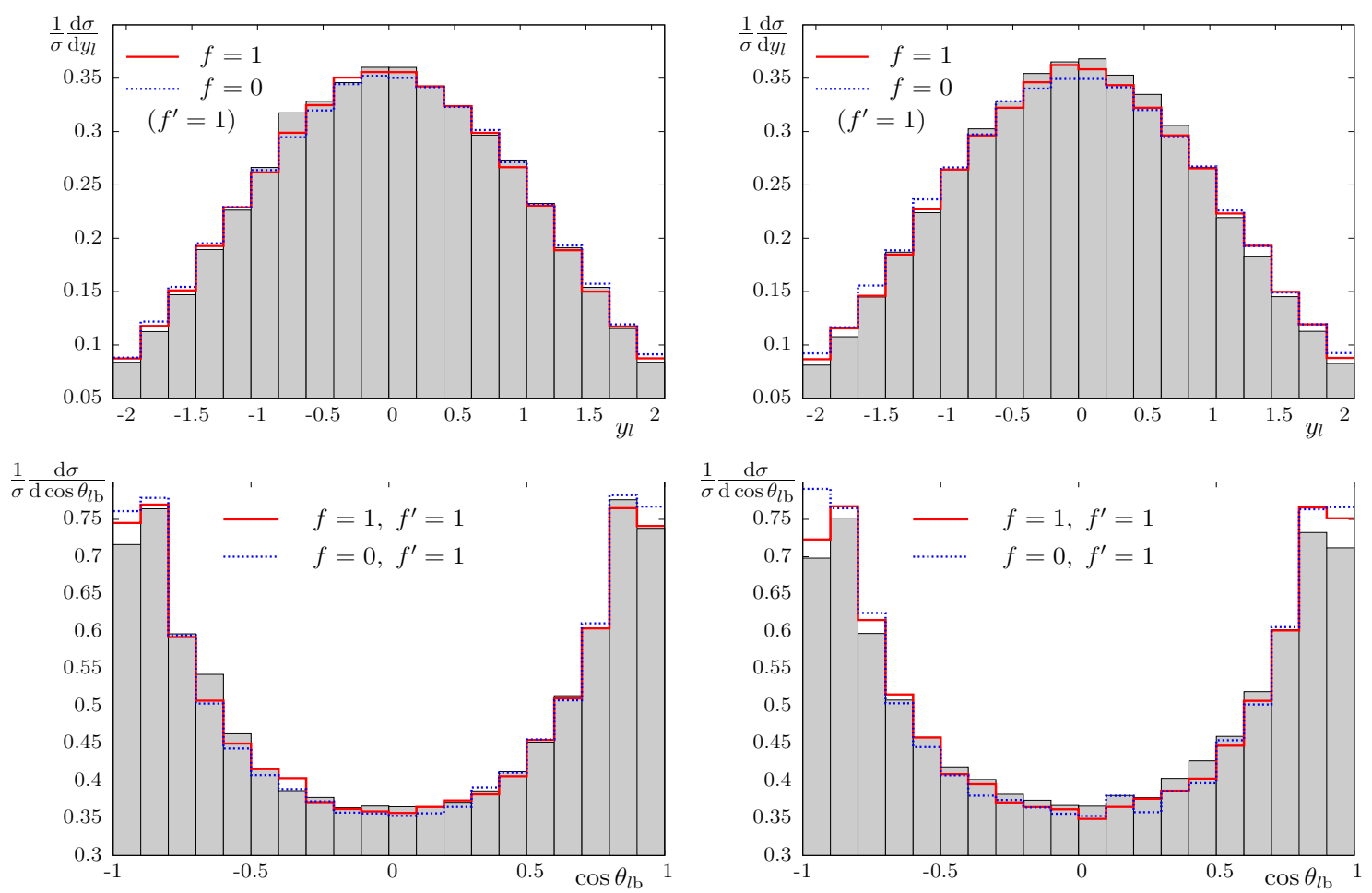

Figure 6. Distributions of the final state lepton of (3.1) at $\sqrt{s}=14 \mathrm{TeV}$ in $y_{l}$ and $\cos \theta_{l \mathrm{~b}}$ with the cuts given by (3.2) (left panels) and (3.2)-(3.6) and (3.7) with $m_{b b}^{\text {cut }}=10 \mathrm{GeV}$ (right panels) for different combinations of the scalar and pseudoscalar tth $h$ couplings.

Open Access. This article is distributed under the terms of the Creative Commons Attribution License which permits any use, distribution and reproduction in any medium, provided the original author(s) and source are credited.

\section{References}

[1] ILC collaboration, J. Brau et al., ILC reference design report: ILC global design effort and world wide study, arXiv:0712.1950 [INSPIRE].

[2] ECFA/DESY LC Physics Working Group collaboration, J. Aguilar-Saavedra et al., TESLA: the superconducting electron positron linear collider with an integrated $x$-ray laser laboratory. technical design report. Part 3. Physics at an $e^{+} e^{-}$linear collider, hep-ph/0106315 [INSPIRE].

[3] American Linear Collider Working Group collaboration, T. Abe et al., Linear collider physics resource book for Snowmass 2001 - Part 2: Higgs and supersymmetry studies, hep-ex/0106056 [INSPIRE].

[4] ACFa Linear Collider Working Group collaboration, K. Abe et al., Particle physics experiments at $J L C$, hep-ph/0109166 [INSPIRE].

[5] CLIC Study, http://clic-study.web.cern.ch/clic-study/.

[6] A. Djouadi, J. Kalinowski and P. Zerwas, Measuring the Htt̄ coupling in $e^{+} e^{-}$collisions, Mod. Phys. Lett. A 7 (1992) 1765 [inSPIRE]. 
[7] A. Djouadi, J. Kalinowski and P. Zerwas, Higgs radiation off top quarks in high-energy $e^{+} e^{-}$ colliders, Z. Phys. C 54 (1992) 255 [InSPIRE].

[8] F.-P. Schilling, Top quark physics at the LHC: a review of the first two years, Int. J. Mod. Phys. A 27 (2012) 1230016 [arXiv:1206.4484] [INSPIRE].

[9] CMS collaboration, First Measurement of the cross section ratio ttbb/ttjj in pp collisions at $\sqrt{s}=7 \mathrm{TeV}$, CMS-PAS-TOP-12-024 (2012).

[10] ATLAS collaboration, Observation of a new particle in the search for the standard model Higgs boson with the ATLAS detector at the LHC, Phys. Lett. B 716 (2012) 1 [arXiv: 1207.7214] [INSPIRE].

[11] CMS collaboration, Observation of a new boson at a mass of $125 \mathrm{GeV}$ with the CMS experiment at the LHC, Phys. Lett. B 716 (2012) 30 [arXiv:1207.7235] [INSPIRE].

[12] K. Kołodziej, 'carlomat', version 2 of the program for automatic computation of lowest order cross sections, arXiv:1305.5096 [INSPIRE].

[13] K. Kołodziej, Carlomat: a program for automatic computation of lowest order cross sections, Comput. Phys. Commun. 180 (2009) 1671 [arXiv:0903.3334] [INSPIRE].

[14] K. Kołodziej, New developments in 'carlomat', Acta Phys. Polon. B 42 (2011) 2477 [arXiv: 1204.5099] [INSPIRE].

[15] J. Aguilar-Saavedra, A minimal set of top-Higgs anomalous couplings, Nucl. Phys. B 821 (2009) 215 [arXiv:0904.2387] [inSPIRE].

[16] K. Kołodziej, Anomalous Wtb coupling at the LHC, arXiv:1212.6733 [INSPIRE].

[17] F. Maltoni, D.L. Rainwater and S. Willenbrock, Measuring the top quark Yukawa coupling at hadron colliders via $t \bar{t} H, H \rightarrow W^{+} W^{-}$, Phys. Rev. D 66 (2002) 034022 [hep-ph/0202205] [INSPIRE].

[18] A. Belyaev and L. Reina, $p p \rightarrow t \bar{t} H, H \rightarrow \tau^{+} \tau^{-}$: toward a model independent determination of the Higgs boson couplings at the LHC, JHEP 08 (2002) 041 [hep-ph/0205270] [INSPIRE].

[19] CMS collaboration, Search for the standard model Higgs boson produced in association with a top-quark pair in pp collisions at the LHC, CMS-HIG-12-035 (2013).

[20] CMS collaboration, Search for the standard model Higgs boson produced in association with a top-quark pair in pp collisions at the LHC, CERN-PH-EP-2013-027 (2013).

[21] CMS collaboration, Search for the standard model Higgs boson produced in association with a top-quark pair in pp collisions at the LHC, JHEP 05 (2013) 145 [arXiv:1303.0763] [INSPIRE].

[22] ATLAS collaboration, Combined coupling measurements of the Higgs-like boson with the ATLAS detector using up to $25 \mathrm{fb}^{-1}$ of proton-proton collision data, ATLAS-CONF-2013-034 (2013).

[23] CMS collaboration, Observation of a new boson with a mass near $125 \mathrm{GeV}$, CMS-PAS-HIG-12-020 (2012).

[24] S. Biswas, E. Gabrielli and B. Mele, Single top and Higgs associated production as a probe of the Htt coupling sign at the LHC, JHEP 01 (2013) 088 [arXiv:1211.0499] [INSPIRE].

[25] S. Biswas, E. Gabrielli, F. Margaroli and B. Mele, Direct constraints on the top-Higgs coupling from the $8 \mathrm{TeV}$ LHC data, arXiv:1304.1822 [INSPIRE]. 
[26] T. Appelquist and M.S. Chanowitz, Unitarity bound on the scale of fermion mass generation, Phys. Rev. Lett. 59 (1987) 2405 [Erratum ibid. 60 (1988) 1589] [INSPIRE].

[27] M. Reece, Vacuum instabilities with a Wrong-sign Higgs-gluon-gluon amplitude, New J. Phys. 15 (2013) 043003 [arXiv:1208.1765] [inSPIRE].

[28] A. Denner, S. Dittmaier, M. Roth and D. Wackeroth, Predictions for all processes $e^{+} e^{-} \rightarrow 4$ fermions $+\gamma$, Nucl. Phys. B 560 (1999) 33 [hep-ph/9904472] [INSPIRE].

[29] A. Denner, S. Dittmaier, M. Roth and D. Wackeroth, RACOONWW1.3: a Monte Carlo program for four fermion production at $e^{+} e^{-}$colliders, Comput. Phys. Commun. 153 (2003) 462 [hep-ph/0209330] [InSPIRE].

[30] J. Pumplin et al., New generation of parton distributions with uncertainties from global QCD analysis, JHEP 07 (2002) 012 [hep-ph/0201195] [INSPIRE].

[31] K. Kolodziej and S. Szczypiński, Off resonance background effects in $e^{+} e^{-} \rightarrow t \bar{t} H$, Nucl. Phys. B 801 (2008) 153 [arXiv:0803.0887] [INSPIRE].

[32] K. Kolodziej and S. Szczypiński, $e^{+} e^{-} \rightarrow t \bar{t} H$ including decays: on the size of background contributions, Eur. Phys. J. C 64 (2009) 645 [arXiv:0903.4606] [INSPIRE].

[33] C. Degrande, J. Gerard, C. Grojean, F. Maltoni and G. Servant, Probing top-Higgs non-standard interactions at the LHC, JHEP 07 (2012) 036 [Erratum ibid. 1303 (2013) 032] [arXiv: 1205.1065] [INSPIRE].

[34] P. Baernreuther, M. Czakon and A. Mitov, Percent level precision physics at the Tevatron: first genuine NNLO QCD corrections to $q \bar{q} \rightarrow t \bar{t}+X$, Phys. Rev. Lett. 109 (2012) 132001 [arXiv: 1204.5201] [INSPIRE]. 